\title{
OPTIMIZATION OF THE LOW LOSS SRF CAVITY FOR THE ILC *
}

\author{
Z. Li ${ }^{1}$, L. Ge, L. Lee, C. Ng, G. Schussman, L. Xiao, K. Ko, SLAC, J. Sekutowicz, DESY, P. \\ Kneisel, TJNAF, T. Higo, Y. Morozumi, K. Saito, KEK
}

Abstract

The Low-Loss shape cavity design has been proposed as a possible alternative to the baseline TESLA cavity design for the ILC main linacs. The advantages of this design over the TESLA cavity are its lower cryogenic loss, and higher achievable gradient due to lower surface fields. High gradient prototypes for such designs have been tested at KEK (ICHIRO) and TJNAF (LL). However, issues related to HOM damping and multipacting still need to be addressed. Preliminary numerical studies of the prototype cavities have shown unacceptable damping factors for some higher-order dipole modes if the typical TESLA HOM couplers are directly adapted to the design. The resulting wakefield will dilute the beam emittance thus reducing the machine luminosity. Furthermore, high gradient tests on a 9-cell prototype at KEK have experienced multipacting barriers although a single LL cell had achieved a high gradient. From simulations, multipacting activities are found to occur in the end-groups of the cavity. In this paper, we will present the optimization results of the end-groups for the Low-Loss designs for effective HOM damping and alleviation of multipacting.

\section{INTRODUCTION}

The Low-Loss (LL) [1] shape cavity design has been proposed as a possible alternative to the baseline TESLA cavity design for the ILC [2] main linacs. The geometry of the LL cavity is optimized to have a lower $B_{\text {peak }} / E_{\text {acc }}$ ratio and a higher shunt $\mathrm{R} / \mathrm{Q}$ by reducing the size of the iris and increasing the cavity volume in the high magnetic field region. As a comparison to the TESLA shape, the iris radius of the LL cell is $30-\mathrm{mm}$, and the side wall of the LL cell is more upright as shown in Fig 1. These modifications resulted in more than $10 \%$ lower in $\mathrm{B}_{\text {peak }} / \mathrm{E}_{\text {acc }}$ and $15 \%$ higher in $\mathrm{R} / \mathrm{Q}$ and geometric factor $\mathrm{G}$ which make the cavity more efficient in acceleration and less cryogenics loss. The maximum gradient achievable in a superconducting cavity is believed to be limited by the critical magnetic flux $B_{c}$ of the niobium which is approximately $180 \mathrm{mT}$ [3]. With the low $\mathrm{B}_{\text {peak }} / \mathrm{E}_{\text {acc }}$, the new shape could support an ultimate gradient of over 50 $\mathrm{MV} / \mathrm{m}$. The realization of such a high gradient design for the ILC linacs would lead to significant cost saving in machine construction and operation. Towards this goal KEK has embarked on a very ambitious schedule to fabricate and test the LL shape cavities (ICHIRO) [4]. At TJNAF, a 7-cell single crystal $\mathrm{Nb}$ prototype [5] is being built and tested to demonstrate performance of the LL shape using a superior superconducting material. Through

\footnotetext{
*Work supported by the U.S. DOE Contract No. DE-AC02-76SF00515.
} 11izh@slac.stanford.edu these tests, a recipe for cavity preparation and treatment is expected to be developed for the high gradient cavity manufacture.

The multipacting (MP) and wakefield damping of the LL cavity are being investigated and optimized through numerical modeling. Preliminary studies of the proposed LL cavity have shown unacceptable damping factors [6] for the high $\mathrm{R} / \mathrm{Q}$ modes in the $3^{\text {rd }}$ dipole band if the typical TESLA HOM couplers are directly adapted to the design. In the high gradient tests of the 9-cell prototype ICHIRO cavity at KEK, strong multipacting barriers were observed at around $30-\mathrm{MV} / \mathrm{m}$ although a single LL cell had achieved a higher gradient. These multipacting activities were identified through simulation to occur in the end-groups. Thus the end-group geometry is crucial for the HOM damping as well as multipacting and need to be addressed in the design and optimization. In this paper, we will present the optimization of the end-groups using Omega3P [7] and Track3P [8] for the Low-Loss designs to effectively damp the HOM modes and to alleviate the multipacting.
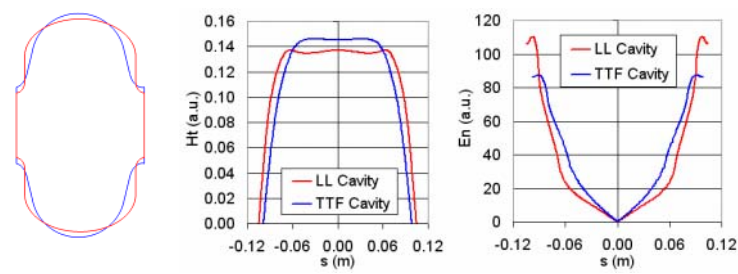

Figure 1: Cavity profile and surface field comparison between Low Loss and TESLA cavity. Red: Low Loss cavity; Blue: TESLA cavity. $\mathrm{S}$ in the field plots is the contour length of the cell from the cell equator.

\section{END GROUP DESIGN CONSIDERATION}

The external Qext of the high R/Q dipole modes need to be damped to bellow the beam instability threshold of $1.0 \times 10^{5}$. The mode spectrum and the $\mathrm{R} / \mathrm{Q}$ values of the $\mathrm{LL}$ design are shown in Fig. 2 for up to the $5^{\text {th }}$ dipole band. The most important modes are the $6 \mathrm{pi} / 9$ mode in the $1^{\text {st }}$ band, $5 \mathrm{pi} / 9$ mode in the $2^{\text {nd }}$ band, and the $0^{\text {th }}$ mode in the third band which has the highest $\mathrm{R} / \mathrm{Q}$. The $4^{\text {th }}$ and $5^{\text {th }}$ dipole bands have relatively lower R/Qs.

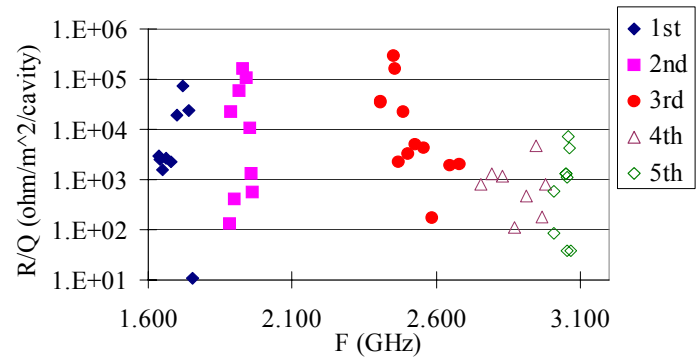

Figure 2: Dipole mode spectrum up to the $5^{\text {th }}$ band for the LL cavity. The highest R/Q modes are in the $3^{\text {rd }}$ band.

T07 Superconducting RF 
The end-group optimization is focused on damping the first 3 dipole bands. The choice of the end beam pipe dimensions is important as the damping design depends on weather the modes propagate or are below cut off in the end pipe. For the modes that propagate in the beam pipes between cavities, the damping is no longer determined by the local HOM couplers alone. In such a case, a multi-cavity system with cavity imperfections needs to be evaluated for the effectiveness of HOM damping. For the LL cavity design, the end beam pipe dimensions are chosen such that the modes up to the $3^{\text {rd }}$ band are cut off. The high R/Q modes are localized in the cavity and can be damped locally by the HOM couplers. This damping scheme is more reliable because the damping is not affected by adjacent cavities. To allow sufficient HOM damping and fundamental mode (FM) coupling, the end beam pipe is composed of a large radius section in the coupler region and then is tapered down to a $30-\mathrm{mm}$ radius which cuts off at $2.9 \mathrm{GHz}$.

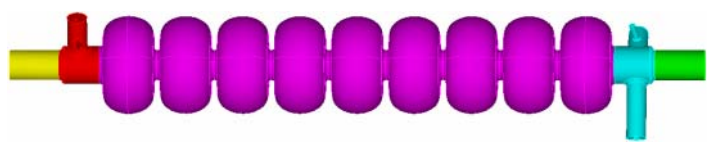

Figure 3: The end beam pipe of the LL cavity has larger radius section for sufficient HOM and FM coupling. The larger radius is tapered down to 30 -mm radius to cut off the modes up to the third band.

The larger end-pipe radius has a significant impact on the third band damping as well as the FM coupling. The required external $\mathrm{Q}_{\mathrm{ext}}$ for a matched FM coupling for a beam current of $11.8 \mathrm{~mA}$ and a gradient of $40 \mathrm{MV} / \mathrm{m}$ is about $3.4 \times 10^{6}$. To obtain such a Qext for the FM mode, the end-pipe radius was initially assumed to be $41 \mathrm{~mm}$ to avoid deep intrusion of the center conductor of the FM coupler into the beam pipe. However, the end-cell with a large beam pipe extension (end-group) could result in a significant difference in frequency between the inner cells and the end-group. Figure 4 shows the difference in the passband frequencies of the inner cell (solid) and the frequencies of the end-group (dashed) for an end-pipe radius of $41-\mathrm{mm}$. At the third band where the modes have the highest $\mathrm{R} / \mathrm{Q}$, the frequency of the end-group is much lower than the inner cells. Thus the modes in the third band are effectively cut off in the end-group, which reduces the field strength in the coupler region and results in poor coupling to the HOM coupler. Preliminary calculations predicted a Qext of $4 \times 10^{5}$ for the high $\mathrm{R} / \mathrm{Q}$ modes in the third band, which is considered not acceptable.

One of the effective ways to increase the field strength in the coupler region (hence improving the HOM coupling) is to use a smaller radius end-pipe to increase the frequency of the end-group to match the frequencies of the inner cells. Figure 5 shows the improvements of the fields in the coupler region with smaller end-pipe radii. At a $38-\mathrm{mm}$ radius, the fields in the coupler region are increased by more than a factor of two while still can provide sufficient FM coupling with an acceptable center conductor intrusion (Fig. 5). The $38-\mathrm{mm}$ pipe radius is chosen for the LL design.

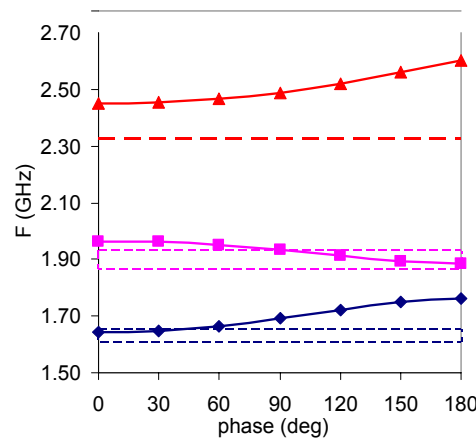

Figure 4: Dipole passbands of the inner cell (solid) and the Frequencies of the end-group (dashed). The frequency of the end-group is much lower than the inner cell at the $3^{\text {rd }}$ passband.
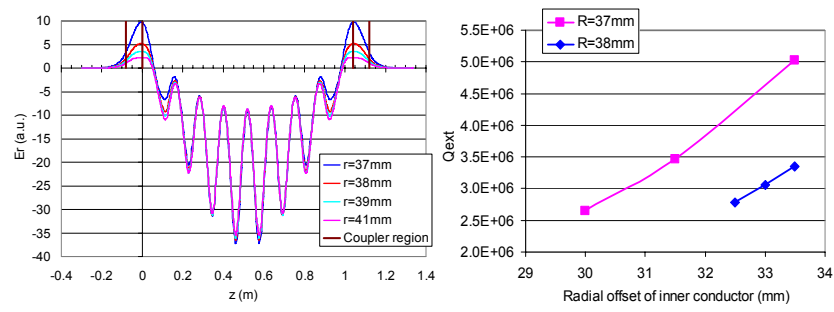

Figure 5: (Left) field enhancement in the coupler region versus end beam pipe radius; (Right) FM coupling versus center conductor position for beam pipe radius of $37-\mathrm{mm}$ and $38-\mathrm{mm}$.

\section{HOM COUPLER OPTIMIZATION}

The HOM couplers for the LL cavity are similar to those of the TESLA cavity. The loop shape and the loop angle were optimized to enhance damping at the $3^{\text {rd }}$ band. Due to asymmetry, both the loop angle and the coupler azimuthal locations have non-negligible perturbations to the dipole mode orientation and were optimized such that the dominate modes are polarized in the $\mathrm{x}$ or y planes. In addition, the azimuthal positions of the HOM couplers were optimized to minimize the coupler kicks due to the fundamental mode.

The loop shape: The nose-tip on the TESLA type coupling loop is eliminated to enhance the magnetic coupling, Fig.6 (a). The width of the loop was reduced to improve the match at the third band frequency.

The loop angle: The loop angle (the angle between the loop plane and the $x-y$ plane) is optimized to couple effectively to the high $\mathrm{R} / \mathrm{Q}$ modes. In the present design, the loop angle is 30 degrees with respect to the $x-y$ plane with the hook side pointing to the cavity as shown in Fig. 6 (b). This angle provides an overall optimal coupling.

The azimuthal location of HOM couplers: In an ideal cavity, the 3D asymmetry of the end-groups affects the polarization of the dipole modes. The azimuthal positions of the HOM couplers and the FM coupler were chosen such that the dominate modes are polarized in the $\mathrm{x}$ or $\mathrm{y}$ 
directions, and the HOM couplers each couples primarily to one of the polarizations. With this arrangement, the HOM couplers will provide similar total coupling to the modes even if they are rotated, as might be the case in actual cavities with imperfections [9]. The HOM coupler at the "downstream" end where the FM coupler is located is positioned to couple to the x-polarization and the HOM coupler on the "upstream" end is positioned to couple only to the y-polarization. The upstream HOM coupler has a 180 degree azimuthal rotational symmetry in terms of the coupling to the y-polarized modes. This provides a freedom to arrange the relative azimuthal positions of the couplers to minimize the coupler kicks due to the fundamental mode. The design for the LL cavity is shown in Fig. 6 (c) where the FM coupler is pointing to the left. Optimized Qext for the first three dipole bands are shown in Fig 7. The Qext of the highest R/Q modes in the third bands is reduced by more than one order of magnitude as compared with simply using the TESLA HOM geometry.

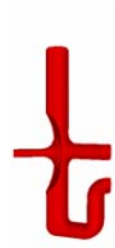

(a)

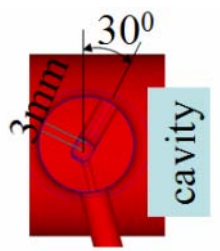

(b)

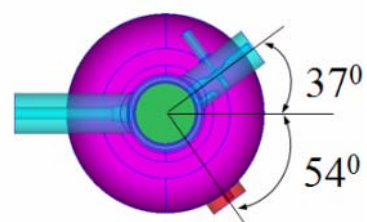

(c)
Figure 6: The optimized end-group and HOM coupler geometries.

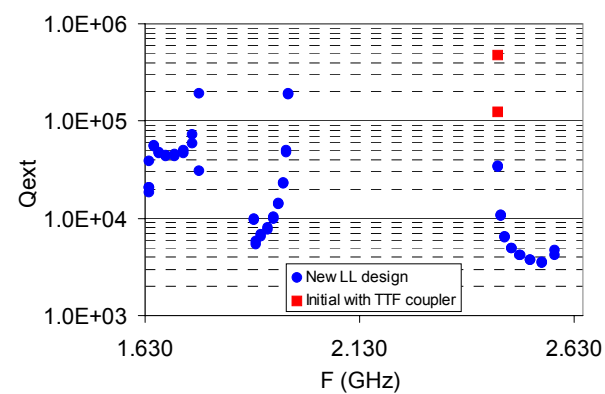

Figure 7: Optimized Qext for the first three dipole bands. The Qext of the highest $\mathrm{R} / \mathrm{Q}$ mode in the $3^{\text {rd }}$ band is $3.4 \times 10^{4}$. The red squares are the Qext with the TESLA HOM coupler for comparison.

\section{MULTIPACING IN THE END-GROUP}

MP in the beam pipe steps: The earlier versions of the ICHIRO cavity suffered multipacting difficulties around $30 \mathrm{MV} / \mathrm{m}$ gradient. These MP barriers were identified by numerical simulations to occur in the region where the beam pipe is tapered from the large radius of 54-mm to $41-\mathrm{mm}$. The cause is due to the combined effect of the step geometry and the strong evanescent fields from the fundamental mode. With the $38-\mathrm{mm}$ end beam pipe radius for the new LL design, the fields are significantly reduced in the $38-30-\mathrm{mm}$ step region as compared with the original ICHIRO design. There was no multipacting observed in the simulation up to $50-\mathrm{MV} / \mathrm{m}$.
MP in the HOM coupler: The first optimized HOM design for the LL cavity showed significant MP activities at field levels starting from $10-\mathrm{MV} / \mathrm{m}$ and up in the gap between the loop and the side wall of the coupler. In Fig. 8 are shown some of the resonant trajectories at $15-\mathrm{MV} / \mathrm{m}$ gradient. The coupling loop in this design has a large flat surface on the side of the gap. This surface and the outer wall of the coupler form a favorable condition for resonant trajectories. To alleviate the multipacting in this gap, the geometry of the hook part of the loop is replaced by a geometry of a circular cross section. The width of the gap is increased to $4.5-\mathrm{mm}$ from $2.5-\mathrm{mm}$. The modified loop design is shown on the right-hand side of Fig. 8 . There was no multipacting found up to $45-\mathrm{MV} / \mathrm{m}$.
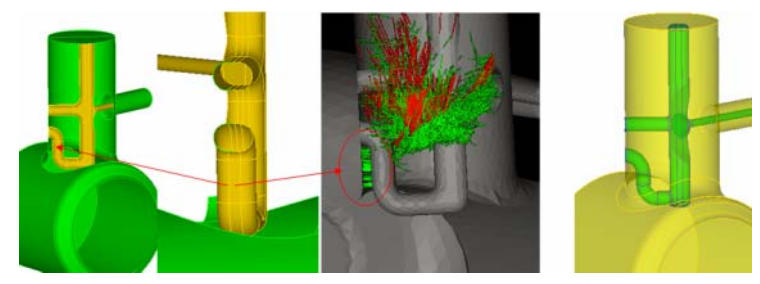

Figure 8: Multipacting in the HOM coupler (left). The multipacting was eliminated by changing the loop geometry from a racetrack cross section to a circular cross section (right).

\section{SUMMARY}

The HOM damping and multipacting were optimized for the LL-shape cavity. Lower than $4 \times 10^{4}$ damping Qext was obtained for the high $\mathrm{R} / \mathrm{Q}$ dipole modes by optimizing the end-group dimensions and coupler loop geometries. The multipacting in the end-group was eliminated in the gradient range of ILC. This design provides a prove of principle damping scheme for the LLshape cavity design to meet the damping requirements for the ILC beam dynamics.

\section{REFERENCES}

[1] J. Sekutowicz et al., "Low Loss Cavity for the $12 \mathrm{GeV}$ CEBAF Upgrade“, JLAB, TN-02-023, June 2002.

[2] http://www.linearcollider.org

[3] K. Saito, "Fundamental RF critical Field Overview," Proc. Workshop on Pushing the Limits of RF Superconductivity, ANL, Argonne, September 2004.

[4] K. Saito et al., proc. of 12th Int. Workshop on RF Superconductivity, Ithaca, NY, July 10-15, 2005.

[5] P. Kneisel et al., proc. of 12th Int. Workshop on RF Superconductivity, Ithaca, NY, July 10-15, 2005.

[6] J. Sekutowicz et al., "Design of a Low Loss SRF Cavity for the ILC," Proc. PAC05, Knoxville, 2005.

[7] L. Lee et al., "Modeling RF Cavity with External Coupling," 2005 SIAM Conference on Computational Science and Engineering, Orlando, Florida, 2005.

[8] L. Ge et al., "Multipacting Simulations of TTF-III Coupler Components," this proceedings.

[9] L. Xiao et al., "TESLA Cavity Imperfection Study," this proceedings. 